\title{
Reaction of Wheat Genotypes to T. laevis Kühn and T. controversa Kühn, the Common and Dwarf Bunts Pathogens in the Dry Highlands of Iran
}

\author{
Kaivan Karimi ${ }^{1}$, Jahanshir Amini ${ }^{2}$, Mahmoud Moradi ${ }^{1}$, Homayoun Kanouni ${ }^{3} \&$ Maghsoud Hassanpour Hosni ${ }^{4}$ \\ ${ }^{1}$ Department of Plant Protection, Agriculture and Natural Resources Research Center of Kurdistan, Sanandaj, Iran \\ ${ }^{2}$ Department of Plant Protection, University of Kurdistan, Sanandaj, Iran \\ ${ }^{3}$ Department of Seed and Plant Improvement, Agriculture and Natural Resources Research Center of Kurdistan, \\ Sanandaj, Iran \\ ${ }^{4}$ Department of Cereal Research, Dryland Agricultural Research Institute (DARI), Maragheh, Iran \\ Correspondence: Jahanshir Amini, Department of plant protection, University of Kurdistan, Sanandaj, Iran. P. O. \\ Box 416. Tel: 98-188-723-563. E-mail: aminij2002@yahoo.com
}

\author{
Received: March 30, 2012 Accepted: April 23, 2012 Online Published: July 11, 2012 \\ doi:10.5539/jas.v4n8p199 URL: http://dx.doi.org/10.5539/jas.v4n8p199
}

\begin{abstract}
Common and dwarf bunts are the most important diseases of wheat in the Kurdistan province, west of Iran. In this study, to investigate the reaction of wheat genotypes (grouped into two cold and warm season genotypes), they were evaluated for infection to common and dwarf bunts during 2007 and 2008 cropping seasons. Plant materials were groups of wheat genotypes included 82 and 158 genotypes from the cold and warm areas which were assessed in this study. For present study, wheat seeds were inoculated by common and dwarf bunts teliospores before planting, and sowing was accomplished during fall season of 2007 and 2008 at Ghamlou and Saral experimental stations. At maturity time, the percentage of infected heads and the main reactions were determined. Results revealed that resistance of genotypes to dwarf bunt was higher than common bunt and also bunt incidence of durum genotypes was relatively lower than bread wheat genotypes. The results demonstrated the existence of resistance gene analogues in durum wheat genotypes which could be used as donor progenitors. Replacement of durum wheat cultivars instead of bread wheat varieties could be an effective way in the developing of substantial farming.
\end{abstract}

Keywords: assessment, common and dwarf bunts, wheat

\section{Introduction}

The entire sown area of wheat in Iran is about 7,035,020 hectares (FAO, 2010), and around 550,057 hectares of land in the Kurdistan province has been allocated to the wheat production. In fact, in this district, wheat is the most important cereal for farmers to food supply and revenue creation. Diseases of wheat mostly caused by fungal pathogens and a few by viruses and bacteria which are important constraints in almost all wheat-growing areas (Rajaram \& van Ginkel, 1996; McIntosh, 1998). In Iran, wheat crop is usually attacked by 15 different diseases at various stages of its growth. Among them, Smuts and rusts are the most important diseases of wheat especially in West and Northwest provinces (Akbari \& Zolghadri, 1988). Although the amount of bunt's damage is less than rust wheat but because of its direct damage to wheat grains, decline in quality and quantity is more tangible.

With regard to history of smut disease, we understand that since the beginnings of wheat cultivation by human bunts have been reduced yield and quality of wheat (Fisher \& Holton, 1957). On the other hand, due to increased wheat monoculture and inappropriate use of seed chemical treatments epidemics have been became more common in small farms.

Among smuts, common and dwarf bunt caused by T. laevis Kühn and T. controversa Kühn are the most serious disease of wheat around the world. Optimum infection of common bunt occurs when teliospore-laden seed is planted in soil at $5^{\circ}$ to $10^{\circ} \mathrm{C}$ (Purdy \& Kendrick, 1963). The distribution and incidence of dwarf bunt is highly 
correlated with snow conditions. Yield significant losses occur only after a winter with an extended period of snow cover in areas where relatively high levels of teliospores are present in the soil (Curtis et al., 2002).

Some studies carried out in evaluation of the different cultivars and lines against common and dwarf bunts and different reactions from genotypes reported by researchers, and a few commercial cultivars also introduced (Kendrik et al., 1957; Knox et al., 1998; Metzger et al., 1977). The extent of yield loss due to common and dwarf bunt in West and Northwest of Iran is estimated about \%30 and \%8.5 respectively, especially when seeds untreated with appropriate fungicide (Asadi \& Behrozin, 1985; Mardoukhi, 1995; Sharifnabi \& Hejadroud, 1992). Common bunt is the most main limitation in successful development of organic winter wheat due to a very limited number of resistant cultivars (Ruzgas \& Liatukas, 2009). The best method to control of bunts especially seed born bunts such as $T$. laevis and $T$. controversa, are using resistant cultivars and fungicides (Dumalasova \& Bartos, 2010). But due to the destructive effects of chemical fungicides on the environment and natural resources, high costs and risk of pathogen resistance to fungicides, its seems that the most optimal way compatible with sustainable agriculture is using of high yielding resistant genotypes, because in addition to being effective in stability of organic agriculture, resistant cultivars are also reduce bunt incidence in the field.

The present study was designed to assess diversity among wheat breeding lines for field resistance. In order to acquiring such a goal, wheat varieties containing cold and warm season genotypes were evaluated against $T$. laevis and T. controversa. The preliminary assessment of wheat lines and their grouping based on their response to disease has been reported in this article.

\section{Materials and Methods}

\subsection{Types of Plant Materials}

Wheat genotypes including 240 lines and cultivars were received through Dryland Agricultural Research Institute (DARI) of Iran; which had selected among advanced nurseries at different cold, dry tropical and subtropical regions. Genotypes of cold zone were including 47 entries from Uniform Regional Wheat Yield Trials (URWYT), 18 from Uniform Regional Durum Yield Trials (URDYT), 14 from Advance Regional Wheat Yield Trials (ARWYT) and three commercial cultivars, and genotypes of warm zone were including 23 genotypes from preliminary yield trials, 19 from Advance Regional Durum Yield Trials (ARDYT), 37 from Elite Regional Wheat Yield Trials (ERWYT) ,59 from URDYT and Uniform Regional Bread Yield Trials (URBYT) as well as three commercial cultivars.

\subsection{Isolation, Inoculation and Assessment of Bunt Incidence}

Table 1. Meteorological details for Ghamlou and Saral experimental stations during crop growing seasons, 2007 and 2008, Kurdistan, Iran

\begin{tabular}{|c|c|c|c|c|c|c|c|c|c|c|c|c|c|c|c|c|}
\hline \multirow{3}{*}{ Month } & \multicolumn{8}{|c|}{ Ghamlou } & \multicolumn{8}{|c|}{ Saral } \\
\hline & \multicolumn{2}{|c|}{ ARH (\%) } & \multicolumn{2}{|c|}{$\mathrm{DB}(0)$} & \multicolumn{2}{|c|}{$\mathrm{AT}$} & \multicolumn{2}{|c|}{$\mathrm{R}$} & \multicolumn{2}{|c|}{ ARH (\%) } & \multicolumn{2}{|c|}{$\mathrm{DB}(0)$} & \multicolumn{2}{|c|}{ AT } & \multicolumn{2}{|c|}{$\mathrm{R}$} \\
\hline & 07-08 & 08-09 & 07-08 & 08-09 & $07-08$ & 08-09 & 07-08 & 08-09 & $07-08$ & 08-09 & $07-08$ & 08-09 & $07-08$ & 08-09 & $07-08$ & 08-09 \\
\hline October & 93.2 & 0 & 13.4 & 12.7 & 0 & 4 & 44 & 34.4 & 78.2 & 0 & 20.08 & 12.83 & 0 & 0 & 57 & 33 \\
\hline November & 48.3 & 3.6 & 3.6 & 6.6 & 18 & 16 & 61.5 & 39.3 & 53.5 & 17.5 & 2.16 & 5.75 & 20 & 11 & 73 & 53 \\
\hline December & 21.1 & 54 & -5.3 & -9 & 31 & 24 & 71.2 & 56.6 & 29 & 37.1 & -4.97 & -2.11 & 30 & 30 & 70 & 72 \\
\hline January & 11.2 & 17.6 & -8.5 & -12 & 30 & 31 & 67.7 & 66.5 & 20.3 & 27.7 & -6.52 & -9.96 & 31 & 31 & 68 & 82 \\
\hline February & 31.9 & 39.7 & 0.2 & -4 & 22 & 24 & 68.2 & 63.8 & 42 & 30.3 & -2.42 & -5.02 & 28 & 29 & 67 & 86 \\
\hline March & 61.3 & 22.9 & 2.7 & 9.4 & 22 & 8 & 57.5 & 36.6 & 47.3 & 19.7 & 0.86 & 6.27 & 29 & 13 & 62.4 & 52 \\
\hline April & 148.7 & 34 & 9.3 & 11.8 & 4 & 4 & 47.5 & 37.4 & 119.3 & 11 & 6.39 & 10.12 & 10 & 3 & 60.36 & 36 \\
\hline May & 39.7 & 2.6 & 15 & 14 & 1 & 2 & 47.2 & 34.5 & 23 & 3 & 13.59 & 10.93 & 0 & 0 & 56 & 33 \\
\hline June & 1.3 & 0 & 18.9 & 19.7 & 0 & 0 & 33 & 28 & 3 & 0 & 18.45 & - & 0 & 0 & 39.55 & 23 \\
\hline July & 2 & 0 & 22.5 & 22.8 & 0 & 0 & 30.2 & 28.2 & 7.5 & 1 & 21.8 & - & 0 & 0 & 35.74 & 21 \\
\hline August & 1.8 & 0 & 22 & 22.4 & 0 & 0 & 30 & 30.0 & 5 & 4.5 & 21.02 & 20.90 & 0 & 0 & 30 & 27 \\
\hline September & 0 & 6.5 & 17.9 & 17.8 & 0 & 0 & 31.4 & 35.4 & 0 & 3 & 19.37 & 18.50 & 0 & 0 & 31.5 & 26 \\
\hline
\end{tabular}

$\mathrm{R}=$ rainfall $(\mathrm{mm}), \mathrm{AT}=$ average temperature $(\mathrm{C}), \mathrm{DB}(0)=$ days below of zero, $\mathrm{ARH}(\%)=$ average relative humidity

Local samples representing $T$. controversa and $T$. laevis were collected from wheat stubble remaining in the fields of Kurdistan province, West of Iran. The seeds of genotypes were artificially inoculated with teliospores of T.laevis and T.controversa; thereby, a high pressure of disease was created through artificial inoculation with bunt inoculums. Teliospores were thoroughly dusted on the wheat seeds just before seeding. Therefore, seeds 
from each genotype were inoculated with spores of fungi in a weighted ratio of five per thousand. Seeds and teliospores mixture were blended for three minutes and sowing was done immediately after the first rainfall. Saral station ( $35^{\circ} 40^{\prime} \mathrm{N}$ lat; $47^{\circ} 07^{\prime} \mathrm{W}$ long; $2120 \mathrm{~m}$ elevation above sea level), where is enough cold and with snow cover (Table 1) was suitable for seeds infected by $T$. controversa and Ghamlou station $\left(35^{\circ} 11^{\prime} \mathrm{N}\right.$ lat; $47^{\circ}$ $29^{\prime} \mathrm{W}$ long and $1867 \mathrm{~m}$ altitude) with normal conditions allocated to study on T. laevis. Entries were sown in adjacent plots of two rows plot ${ }^{-1}$; with each row of $1 \mathrm{~m}$ long and $0.25 \mathrm{~m}$ apart. Sampling and calculating of healthy and infected heads were rated in July. The disease status was examined visually and the severity of disease was presented as percentage for bunt disease. The percentage of infected heads index calculated according to Mamlouk and Van Slageren (1993). Based on this recommended index, samples up to 5\% infected heads categorized as resistance $(\mathrm{R}=1)$, more than $5 \%$ and less or equal $10 \%$ as moderately resistant $(\mathrm{MR}=3)$, $10 \%$ and less or equal $15 \%$ as mediate susceptible $(\mathrm{MS}=5)$, and infected above $15 \%$ as susceptible $(\mathrm{S}=7)$, in here, odd numbers 1 to 7 was given to indices for calculation of statistical computations.

Incidence percent of disease was calculated by:

$$
\mathrm{W}=(\mathrm{A} / \mathrm{B}) \times 100
$$

Where, $\mathrm{A}=$ infected heads and, $\mathrm{B}=$ total of evaluated heads.

\subsection{Statistical Analysis}

This study consisted of 240 wheat genotypes with small seed amount, each sown in two rows without replications, during two successive years. The differences between disease score of $T$. laevis and $T$. controversa on bread and durum wheats were assessed with paired $t$-tests after appropriate transformations. Data sets for bread and durum wheat germplasms on $T$. laevis and on $T$. controversa were also analysed using $t$-test with unequal variance assumption.

\section{Results}

\subsection{Reactions to T. laevis during 2007 and 2008}

In 2007, from 47 entries adapted to cold regions, 10 revealed resistance. Among the ARWYT's entries, No. 56 with $6 \%$ infection was partially resistant and 13 lines with a high intensity of infection were susceptible to the disease. Among the 18 lines of URDYT, two were susceptible and 16 lines were mostly infection free and showed considerable resistance. Zardak, cold commercial cultivar, was resistant without any infection, Sardari was partially resistant and Azar2 was susceptible with $18 \%$ of infection. From lines related to the preliminary yield trials, 12 were resistant. From 59 lines of sets URBYT and URDYT, 22 lines were resistant. Among 37 lines of ERWYT, 33 were mostly non-affected. The results also showed that among 19 ARDYT varieties, 17 with low symptoms classified as resistant to disease. Warm commercial cultivars showed different reactions, thus Saimarae and Gahar were resistant, Kouhdasht and Cham-4 partially resistant and, Pastor and Zagros represented susceptible reaction (Table 2).

Table 2. Reaction of wheat genotypes to common and dwarf bunts during two successive cropping seasons, 2007-08 and 2008-09 at Kurdistan, Iran

\begin{tabular}{|c|c|c|c|c|c|c|c|c|c|c|}
\hline \multirow[t]{3}{*}{ Genotypes } & \multicolumn{5}{|c|}{ T. laevis } & \multicolumn{5}{|c|}{ T. controversa } \\
\hline & \multicolumn{2}{|c|}{2007} & \multicolumn{2}{|c|}{2008} & \multirow[b]{2}{*}{ MDI } & \multicolumn{2}{|c|}{2007} & \multicolumn{2}{|c|}{2008} & \multirow[b]{2}{*}{ MDI } \\
\hline & PI & DI & PI & DI & & PI & DI & PI & DI & \\
\hline UNKN/HATUSHA//BEZ/.....B & 35 & $\mathrm{~S}$ & 16 & $\mathrm{~S}$ & $\mathrm{~S}$ & 2 & $\mathrm{R}$ & 0 & $\mathrm{R}$ & $\mathrm{R}$ \\
\hline BJN C 79/4/KVZ/CUT75/3/YMH/ ...B & 11 & MS & 6 & MR & MR & 3 & $\mathrm{R}$ & 0 & $\mathrm{R}$ & $\mathrm{R}$ \\
\hline FKG 13/4/NWT/3/TAST/SPRW/.. B & 12 & MS & 2 & $\mathrm{R}$ & MR & 3 & $\mathrm{R}$ & 0 & $\mathrm{R}$ & $\mathrm{R}$ \\
\hline IG42650/6/ZCL/3/PGFN//...B & 15 & MS & 4 & $\mathrm{R}$ & MR & 0 & $\mathrm{R}$ & 0 & $\mathrm{R}$ & $\mathrm{R}$ \\
\hline Tam 200/Kauz...B & 27 & S & 7 & MR & S & 2 & $\mathrm{R}$ & 0 & $\mathrm{R}$ & $\mathrm{R}$ \\
\hline 130L1.11//F35.70/Mo73/...B & 30 & S & 3 & $\mathrm{R}$ & $\mathrm{S}$ & 6 & MR & 0 & $\mathrm{R}$ & $\mathrm{R}$ \\
\hline Ok84306//Cno79/Prl/3/...B & 65 & $\mathrm{~S}$ & 30 & $\mathrm{~S}$ & S & 12 & MS & 0 & $\mathrm{R}$ & MR \\
\hline Bayrak tar.. B & 8 & MR & 8 & MS & MR & 1 & $\mathrm{R}$ & 0 & $\mathrm{R}$ & $\mathrm{R}$ \\
\hline 96 Gen bank 82-maragheh... B & 3 & $\mathrm{R}$ & 1 & $\mathrm{R}$ & $\mathrm{R}$ & 6 & MR & 0 & $\mathrm{R}$ & $\mathrm{R}$ \\
\hline $\begin{array}{l}\text { TAST/TORIM/3/MLC/4/CWW339.5/SP } \\
\text { N/... B }\end{array}$ & 6 & MR & 3 & $\mathrm{R}$ & $\mathrm{R}$ & 9 & MR & 0 & $\mathrm{R}$ & $\mathrm{R}$ \\
\hline TIRCHMIR1/LCO//CA 8055/9/P ... B & 2 & $\mathrm{R}$ & 3 & $\mathrm{R}$ & $\mathrm{R}$ & 7 & MR & 0 & $\mathrm{R}$ & $\mathrm{R}$ \\
\hline GB-SARA-27- B & 20 & $\mathrm{~S}$ & 3 & $\mathrm{R}$ & MR & 8 & MR & 0 & $\mathrm{R}$ & $\mathrm{R}$ \\
\hline
\end{tabular}


GB-SARA-244- B

Shahi/T-C(22) IRW-MR- B

SPII BWSP(F5 : 3)-7MAR-

BJN837/GRK//HK47 ICWH99276.. B

BJN837/GRK//HK47 ICWH99276.. B

GRK/5/RRV/WW15/3/BJ/2*ON//BON.

ARG/R16//BEZ*2/3/AGRI/KSK/5/TRK

13/6/M ICWH99324.. B

TRK13/4/SNB/HN4//SPN/3/WTS//YM

H/HYS/5 ICWH99365.. B

OR

F1.158/FDL//BLO/3/SHI4414/CROW/4/

C ICWH99381.. B

SARDARI-HD35/5/DMN//SUT/AG(ES

86-7)/3/

ICWH99-0551-0AP-0AP-0AP-OMAR-7

MAR B

SARDARI-HD35/5/DMN//SUT/AG(ES

86-7)/3/

ICWH99-0552-0AP-0AP-0AP-OMAR-3

MAR- B

SARDARI-HD35/SARDARI//PRINIA

ICWH99..B

SARDARI-HD74//LINFEN875072/KA

UZ/6/VEE

ICWH99-0596-0AP-0AP-0AP-OMAR-6

MAR- B

SARDARI-HD83//PTZ

NISKA/UT1556-170/3/

ICWH99-0600-0AP-0AP-0AP-OMAR-1

MAR- B

L 44-29 K

4-1/4/RPB868/CHRC//UT1567.1

ICWH99-0618-0AP-0AP-0AP-OMAR-1

MAR- B

L 44-29 K

4-1/4/RPB868/CHRC//UT1567.1

ICWH99-0618-0AP-0AP-0AP-OMAR-3

MAR- B

90ZHONG65/4/CO724377/NAC//SERI/

3/TRK1.. B

YUMAI13/5/NAI60/3/14.53/ODIN//CI1

3441.. B

shi $\neq 4414 /$ crow"s"//Attila.. B

shi $\neq 4414 /$ crow"s"//WW33/Vee"s"- B

Vee"s"/Nac//shi $\neq 4414 /$ crow"s"-

885K4.1//MNG/sDv1/3/1d13.1/MLT- B

TRILIA/MV17

TCI951373-0SE-0YC-0E-1YE-0YE-3Y

M- B

ISWYN32/3/DONG87//TJB368.251/BU

C/4/MV17

TCI952176-0SE-0YC-0E-6YE- B

AGRI/BJY//VEE/3/KRC66(excellentsee d) TCI951025-0SE-0YC-0E-2YE-0YE PONY/OPATA/5/CA8055/4/ROMTAS

$\begin{array}{cccccccccc}4 & \mathrm{R} & 6 & \mathrm{MR} & \mathrm{R} & 3 & \mathrm{R} & 0 & \mathrm{R} & \mathrm{R} \\ 10 & \mathrm{MR} & 12 & \mathrm{MS} & \mathrm{MS} & 6 & \mathrm{MR} & 0 & \mathrm{R} & \mathrm{R} \\ 3 & \mathrm{R} & 1 & \mathrm{R} & \mathrm{R} & 5 & \mathrm{R} & 0 & \mathrm{R} & \mathrm{R} \\ 5 & \mathrm{R} & 4 & \mathrm{R} & \mathrm{R} & 5 & \mathrm{R} & 0 & \mathrm{R} & \mathrm{R} \\ 12 & \mathrm{MS} & 20 & \mathrm{~S} & \mathrm{~S} & 2 & \mathrm{R} & 0 & \mathrm{R} & \mathrm{R} \\ 37 & \mathrm{~S} & 2 & \mathrm{R} & \mathrm{S} & 20 & \mathrm{~S} & 0 & \mathrm{R} & \mathrm{MR} \\ 4 & \mathrm{R} & 4 & \mathrm{R} & \mathrm{R} & 3 & \mathrm{R} & 0 & \mathrm{R} & \mathrm{R} \\ & & & & & & & & & \\ 24 & \mathrm{~S} & 12 & \mathrm{MS} & \mathrm{S} & 21 & \mathrm{~S} & 0 & \mathrm{R} & \mathrm{MS} \\ & & & & & & & & & \\ 22 & \mathrm{~S} & 16 & \mathrm{~S} & \mathrm{~S} & 11 & \mathrm{MS} & 0 & \mathrm{R} & \mathrm{MR}\end{array}$

$\begin{array}{lllllllllll}8 & \text { MR } & 7 & \text { MR } & \text { MR } & 9 & \text { MR } & 0 & \text { R } & \text { R }\end{array}$ $\begin{array}{llllllllllll}5 & \mathrm{R} & 4 & \mathrm{R} & \mathrm{R} & 2 & \mathrm{R} & 0 & \mathrm{R} & \mathrm{R}\end{array}$ $\begin{array}{lllllllllllllllll}2 & \mathrm{R} & 0 & \mathrm{R} & \mathrm{R} & 1 & \mathrm{R} & 0 & \mathrm{R} & \mathrm{R}\end{array}$ $\begin{array}{lllllllllll}38 & \mathrm{~S} & 5 & \mathrm{R} & \mathrm{S} & 35 & \mathrm{~S} & 0 & \mathrm{R} & \mathrm{S}\end{array}$ $\begin{array}{lllllllllll}4 & \mathrm{R} & 4 & \mathrm{R} & \mathrm{R} & 6 & \mathrm{MR} & 0 & \mathrm{R} & \mathrm{R}\end{array}$ $\begin{array}{lllllllllll}6 & \mathrm{MR} & 5 & \mathrm{R} & \mathrm{MR} & 10 & \mathrm{MR} & 0 & \mathrm{R} & \mathrm{R}\end{array}$ $\begin{array}{lllllllllll}6 & \text { MR } & 100 & \mathrm{~S} & \mathrm{~S} & 9 & \mathrm{MR} & 0 & \mathrm{R} & \mathrm{R}\end{array}$ $\begin{array}{lllllllllll}24 & \mathrm{~S} & 3 & \mathrm{R} & \mathrm{MS} & 12 & \mathrm{MS} & 0 & \mathrm{R} & \mathrm{MR}\end{array}$ $\begin{array}{llllllllllll}4 & \mathrm{R} & 1 & \mathrm{R} & \mathrm{R} & 4 & \mathrm{R} & 0 & \mathrm{R} & \mathrm{R}\end{array}$ $\begin{array}{llllllllll}38 & \mathrm{~S} & 20 & \mathrm{~S} & \mathrm{~S} & 28 & \mathrm{~S} & 0 & \mathrm{R} & \mathrm{MS}\end{array}$ $\begin{array}{llllllllll}13 & \mathrm{MS} & 2 & \mathrm{R} & \mathrm{MR} & 16 & \mathrm{~S} & 0 & \mathrm{R} & \mathrm{MR}\end{array}$ $\begin{array}{llllllllll}62 & \mathrm{~S} & 7 & \mathrm{MR} & \mathrm{S} & 17 & \mathrm{~S} & 0 & \mathrm{R} & \mathrm{MR}\end{array}$ $\begin{array}{llllllllll}11 & \mathrm{MS} & 11 & \mathrm{MS} & \mathrm{MS} & 3 & \mathrm{R} & 0 & \mathrm{R} & \mathrm{R}\end{array}$ $\begin{array}{lllllllllllll}2 & \mathrm{R} & 1 & \mathrm{R} & \mathrm{R} & 1 & \mathrm{R} & 0 & \mathrm{R} & \mathrm{R}\end{array}$ $\begin{array}{llllllllll}17 & \mathrm{~S} & 7 & \mathrm{MR} & \mathrm{MS} & 16 & \mathrm{~S} & 0 & \mathrm{R} & \mathrm{MR}\end{array}$ $\begin{array}{lllllllllll}58 & \mathrm{~S} & 15 & \mathrm{MS} & \mathrm{S} & 6 & \mathrm{MR} & 0 & \mathrm{R} & \mathrm{R}\end{array}$ $\begin{array}{lllllllllll}57 & \mathrm{~S} & 4 & \mathrm{R} & \mathrm{S} & 37 & \mathrm{~S} & 0 & \mathrm{R} & \mathrm{S}\end{array}$ 
T/BON/3/DIBO//SU92/CI13645

TCI950094-22AP-0AP-0E-1YE-0YE-2

YM-0YM- B

YE2453//PPBB68/CHRC

TCI950019-8AP-0AP-0E-3YE- B

HARTOG

CM8399-D-4M-4Y-2M-2Y-0M- B

TUICM74849-2M-3Y-1M-1Y-1M-0Y-

\section{B}

ATTILA//AGRI/NAC- B

RINA-11- B

BATAVIA/TAMEX/OPATA/3/ID8009

94.W/VEE- B

VRZ/3/ORF1.148/TDL//BLO/4/KS82W

409/STP- B

PONY/OPATA/5/CA8055/4/ROMTAS

T/BON/3/DIBO//SU92/CI13645- B

AGRI/BJY//VEE/3/PRINIA- B

CA8055/4/ROMTAST/BON/3/DIBO//S

U92/CI13645/5/AGRI/BJY//VEES- B

PONY/OPATA/5/CA8055/4/ROMTAS

T/BON/3/DIBO//SU92/CI13645- B

SHI\#4414/CROWS"//UNKNOWN- B 3

ERYT5678-87/F900K//SULTAN95- B

SABALAN/4/VRZ/3/OR

F1.148/TDL//BLO- B

MAHON

DEMIAS/3/HIM/CNDR//CA8055- B

AGRI/NAC//LIRA/3/PONY/OPATA- B

F134.71/NAC//ZOMBOR- B

KS82W409/STEPHENS/3/TAST/PCH//

BEZ... B

ZCL/3/PGFN//CNO67/SON64(ES86-8)/

4/SERI/5/UA-2837- B

$\begin{array}{llllllllll}84 & \mathrm{~S} & 60 & \mathrm{~S} & \mathrm{~S} & 65 & \mathrm{~S} & 0 & \mathrm{R} & \mathrm{S}\end{array}$

$\begin{array}{lllllllllll}34 & \mathrm{~S} & 20 & \mathrm{~S} & \mathrm{~S} & 8 & \mathrm{MR} & 0 & \mathrm{R} & \mathrm{R}\end{array}$

$\begin{array}{llllllllll}22 & \mathrm{~S} & 2 & \mathrm{R} & \mathrm{MS} & 21 & \mathrm{~S} & 0 & \mathrm{R} & \mathrm{MS}\end{array}$

$\begin{array}{llllllllll}49 & \mathrm{~S} & 1 & \mathrm{R} & \mathrm{S} & 3 & \mathrm{R} & 0 & \mathrm{R} & \mathrm{R}\end{array}$

$\begin{array}{llllllllll}9 & \mathrm{MR} & 2 & \mathrm{R} & \mathrm{MR} & 0 & \mathrm{R} & 0 & \mathrm{R} & \mathrm{R}\end{array}$

$\begin{array}{llllllllll}88 & \mathrm{~S} & 20 & \mathrm{~S} & \mathrm{~S} & 16 & \mathrm{~S} & 0 & \mathrm{R} & \mathrm{MR}\end{array}$

$\begin{array}{lllllllllll}30 & \mathrm{~S} & 12 & \mathrm{MS} & \mathrm{S} & 22 & \mathrm{~S} & 0 & \mathrm{R} & \mathrm{MS}\end{array}$

$\begin{array}{llllllllll}66 & \mathrm{~S} & 25 & \mathrm{~S} & \mathrm{~S} & 25 & \mathrm{~S} & 0 & \mathrm{R} & \mathrm{MS}\end{array}$

$\begin{array}{llllllllll}30 & \mathrm{~S} & 20 & \mathrm{~S} & \mathrm{~S} & 16 & \mathrm{~S} & 0 & \mathrm{R} & \mathrm{MS}\end{array}$

$\begin{array}{llllllllll}55 & \mathrm{~S} & 8 & \mathrm{MR} & \mathrm{S} & 8 & \mathrm{MR} & 0 & \mathrm{R} & \mathrm{R}\end{array}$

$\begin{array}{lllllllllll}90 & \mathrm{~S} & 50 & \mathrm{~S} & \mathrm{~S} & 18 & \mathrm{~S} & 0 & \mathrm{R} & \mathrm{MR}\end{array}$

$\begin{array}{llllllllll}39 & \mathrm{~S} & 64 & \mathrm{~S} & \mathrm{~S} & 9 & \mathrm{MR} & 0 & \mathrm{R} & \mathrm{R}\end{array}$

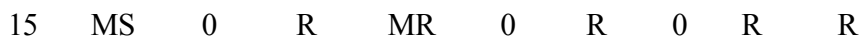

$\begin{array}{lllllllllll}17 & \mathrm{~S} & 1 & \mathrm{R} & \mathrm{MR} & 4 & \mathrm{R} & 0 & \mathrm{R} & \mathrm{R}\end{array}$

$\begin{array}{lllllllllll}25 & \mathrm{~S} & 10 & \mathrm{MR} & \mathrm{S} & 0 & \mathrm{R} & 0 & \mathrm{R} & \mathrm{R}\end{array}$

$\begin{array}{llllllllll}24 & \mathrm{~S} & 20 & \mathrm{~S} & \mathrm{~S} & 2 & \mathrm{R} & 0 & \mathrm{R} & \mathrm{R}\end{array}$

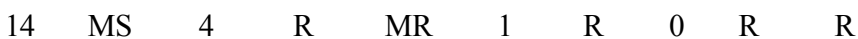

$\begin{array}{llllllllll}6 & \mathrm{MR} & 30 & \mathrm{~S} & \mathrm{~S} & 0 & \mathrm{R} & 0 & \mathrm{R} & \mathrm{R}\end{array}$

$\begin{array}{lllllllllll}24 & \mathrm{~S} & 42 & \mathrm{~S} & \mathrm{~S} & 3 & \mathrm{R} & 0 & \mathrm{R} & \mathrm{R}\end{array}$

ORE

F1.158/FDL//BLO/3/SHI4414/CROW/4/

SABALAN- B

SHI\#4414/CROWS"//UNKNOWN- B

VRZ/3/OR

F1 158/FDL//BLO/4/VRZ/3/OR

F1.148/TDL//B - B

DARI-16//KS82W409/STEPHENS- B

44-16-2-4-D

25-25-1-5- D

40-11-2-3- D

20-16-1-4- D

18-18-1-4- D

74-23-3-5 - D

73-16-3-5- D

29-18-2-1- D

71-7-3-5- D

57-11-3-1- D

43-25-2-4- D

19-17-1-4- D

409- D

42- D $\begin{array}{llllllllll}12 & \text { MS } & 15 & \text { MS } & \text { MS } & 3 & \text { R } & 0 & \text { R } & \text { R }\end{array}$

$\begin{array}{lllllllllll}44 & \mathrm{~S} & 30 & \mathrm{~S} & \mathrm{~S} & 7 & \mathrm{MR} & 0 & \mathrm{R} & \mathrm{R}\end{array}$

$\begin{array}{llllllllllllllll}21 & \mathrm{~S} & 25 & \mathrm{~S} & \mathrm{~S} & 2 & \mathrm{R} & 0 & \mathrm{R} & \mathrm{R}\end{array}$

$\begin{array}{cccccccccc}16 & \mathrm{~S} & 50 & \mathrm{~S} & \mathrm{~S} & 4 & \mathrm{R} & 0 & \mathrm{R} & \mathrm{R} \\ 0 & \mathrm{R} & 1 & \mathrm{R} & \mathrm{R} & 0 & \mathrm{R} & 0 & \mathrm{R} & \mathrm{R} \\ 1 & \mathrm{R} & 0 & \mathrm{R} & \mathrm{R} & 0 & \mathrm{R} & 0 & \mathrm{R} & \mathrm{R} \\ 0 & \mathrm{R} & 0 & \mathrm{R} & \mathrm{R} & 0 & \mathrm{R} & 0 & \mathrm{R} & \mathrm{R} \\ 0 & \mathrm{R} & 0 & \mathrm{R} & \mathrm{R} & 0 & \mathrm{R} & 0 & \mathrm{R} & \mathrm{R} \\ 0 & \mathrm{R} & 0 & \mathrm{R} & \mathrm{R} & 0 & \mathrm{R} & 0 & \mathrm{R} & \mathrm{R} \\ 0 & \mathrm{R} & 0 & \mathrm{R} & \mathrm{R} & 0 & \mathrm{R} & 0 & \mathrm{R} & \mathrm{R} \\ 0 & \mathrm{R} & 0 & \mathrm{R} & \mathrm{R} & 1 & \mathrm{R} & 0 & \mathrm{R} & \mathrm{R} \\ 0 & \mathrm{R} & 0 & \mathrm{R} & \mathrm{R} & 1 & \mathrm{R} & 0 & \mathrm{R} & \mathrm{R} \\ 0 & \mathrm{R} & 0 & \mathrm{R} & \mathrm{R} & 1 & \mathrm{R} & 0 & \mathrm{R} & \mathrm{R} \\ 1 & \mathrm{R} & 0 & \mathrm{R} & \mathrm{R} & 0 & \mathrm{R} & 0 & \mathrm{R} & \mathrm{R} \\ 0 & \mathrm{R} & 0 & \mathrm{R} & \mathrm{R} & 0 & \mathrm{R} & 0 & \mathrm{R} & \mathrm{R} \\ 0 & \mathrm{R} & 0 & \mathrm{R} & \mathrm{R} & 5 & \mathrm{R} & 0 & \mathrm{R} & \mathrm{R} \\ 2 & \mathrm{R} & 0 & \mathrm{R} & \mathrm{R} & 0 & \mathrm{R} & 0 & \mathrm{R} & \mathrm{R} \\ 1 & \mathrm{R} & 0 & \mathrm{R} & \mathrm{R} & 0 & \mathrm{R} & 0 & \mathrm{R} & \mathrm{R}\end{array}$




\section{8- D}

Gcn//Stj/Mrb3- D

Ch1/Brach//Mra-I- D

Lgt3/4/Bcr/3/Ch1//Gta/Stk- D

Zardak-B

Sradari-B

Azar-2-B

$\mathrm{KAUZ} / \mathrm{BOW} / \mathrm{NKT}$

$\begin{array}{lllllllllll}\text { CMSS92Y02933S-20Y-015M-010Y-010 } & 12 & \text { MS } & 8 & \text { R } & \text { MR } & 3 & \text { R } & 0 & \text { R } & \text { R }\end{array}$

Y-8M-0Y-0AP-B

SW89.5181/KAUZ

$\begin{array}{lllllllllll}\text { CMSS93B00824S-24Y-010M-010Y-010 } & 24 & \mathrm{~S} & 20 & \mathrm{~S} & \mathrm{~S} & 9 & \mathrm{MR} & 0 & \mathrm{R} & \mathrm{R}\end{array}$ M-9Y-0M-0AP-B

KAUZ/FLORKWA-1

$\begin{array}{llllllllllll}\text { ICW97-0444-0APS-5AP-0APS-050AP- } & 2 & \mathrm{R} & 3 & \mathrm{R} & \mathrm{R} & 11 & \mathrm{MS} & 0 & \mathrm{R} & \mathrm{MR}\end{array}$ 0APS-26AP-0AP- B

YMI\#6/GEN//TIA.1/3/VEE\#5//DOVE/B

$\mathrm{UC}$

CMS93Y00810S-040M-0100Y-0150Y-0

40M-020Y-0M-33Y-OM-- B

CROC-1/AE.SQUARROSA

(205)//KAUZ/3/SASIACMSS93Y01026

S-040M- B

SHUHA-7//SERI 82/SHUHA 'S

ICW97-0137-7AP-0APS-8AP_0APS-03

0AP-0AP- B

SW89.2089/KAUZ

$\begin{array}{lllllllllll}\text { CMSS93B00870S-2Y-010M-010Y-010 } & 4 & \text { R } & 8 & \text { MR } & \text { MR } & 1 & \text { R } & 0 & \text { R } & \text { R }\end{array}$

M-7Y-0M-6M-0Y- B

SW89.5193/KAUZ

$\begin{array}{llllllllllll}\text { CMSS94Y00761S-0300M-0100Y-0100 } & 82 & \mathrm{~S} & 40 & \mathrm{~S} & \mathrm{~S} & 2 & \mathrm{R} & 0 & \mathrm{R} & \mathrm{R}\end{array}$

M-18Y-9M-0Y- B

SERI*3//RL601 0/4*YR/3/P

ASTOR/4/BA V92

CMSS96M05696T-040Y-14M-O1

OSY-O1 OM-O1 OSY-4M- OY- B

KARAJ 83- 1062- B

KARAJ 83- 1272- B

KARAJ 83- 1316- B

NANJING82149/KAUZ/3/PFAU/SERI//

BOW- B

INQALAB 91 PB19545-9A-0A-0PAK-

B

PBW343

CM85836-4Y-OM-OY-8M-OY-OIND-

ITAPUA 40-OBLIGADO

CM9493-3M-2Y-5M-1Y-0M-0PRY- B PBW343

CM85836-4Y-0M-0Y-8M-0Y-0IND- B

SRMA/TUI//PASDTOR $\begin{array}{llllllllllll}\text { CMSS97M00386S-40M-040SY-030M-0 } & 15 & \text { MS } & 20 & \text { S } & \text { S } & 2 & \text { R } & 0 & \text { R } & \text { R }\end{array}$ 40SY-33M-0Y-0SY- B

SLVS/PASTOR

CMSS97Y04126S-5Y-010M-010SY-01 32

OM-8SY-010M- B

$\begin{array}{lllllllllll}44 & \mathrm{~S} & 15 & \mathrm{MS} & \mathrm{S} & 0 & \mathrm{R} & 0 & \mathrm{R} & \mathrm{R}\end{array}$

$\begin{array}{llllllllll}3 & \mathrm{R} & 2 & \mathrm{R} & \mathrm{R} & 9 & \mathrm{MR} & 0 & \mathrm{R} & \mathrm{R}\end{array}$

$\begin{array}{llllllllll}20 & \mathrm{~S} & 14 & \mathrm{MS} & \mathrm{S} & 0 & \mathrm{MR} & 0 & \mathrm{R} & \mathrm{R}\end{array}$

$\begin{array}{llllllllll}1 & \mathrm{R} & 0 & \mathrm{R} & \mathrm{R} & 3 & \mathrm{R} & 0 & \mathrm{R} & \mathrm{R}\end{array}$

$\begin{array}{llllllllll}0 & \mathrm{R} & 0 & \mathrm{R} & \mathrm{R} & 1 & \mathrm{R} & 0 & \mathrm{R} & \mathrm{R}\end{array}$

$\begin{array}{llllllllll}7 & \mathrm{MR} & 2 & \mathrm{R} & \mathrm{R} & 0 & \mathrm{R} & 0 & \mathrm{R} & \mathrm{R}\end{array}$

$\begin{array}{llllllllll}18 & \mathrm{~S} & 17 & \mathrm{~S} & \mathrm{~S} & 0 & \mathrm{R} & 0 & \mathrm{R} & \mathrm{R}\end{array}$ $\begin{array}{llllllllllll}3 & \mathrm{R} & 3 & \mathrm{R} & \mathrm{R} & 1 & \mathrm{R} & 0 & \mathrm{R} & \mathrm{R}\end{array}$ $\begin{array}{llllllllll}7 & \mathrm{MR} & 7 & \mathrm{MR} & \mathrm{MR} & 0 & \mathrm{R} & 0 & \mathrm{R} & \mathrm{R}\end{array}$ $\begin{array}{llllllllllll}6 & \mathrm{MR} & 3 & \mathrm{R} & \mathrm{R} & 1 & \mathrm{R} & 0 & \mathrm{R} & \mathrm{R}\end{array}$ $\begin{array}{llllllllllll}78 & \mathrm{~S} & 35 & \mathrm{~S} & \mathrm{~S} & 5 & \mathrm{R} & 0 & \mathrm{R} & \mathrm{R}\end{array}$ $\begin{array}{lllllllllll}9 & \mathrm{MR} & 40 & \mathrm{~S} & \mathrm{~S} & 4 & \mathrm{R} & 0 & \mathrm{R} & \mathrm{R}\end{array}$ $\begin{array}{llllllllll}29 & \mathrm{~S} & 15 & \mathrm{MS} & \mathrm{S} & 10 & \mathrm{MR} & 0 & \mathrm{R} & \mathrm{R}\end{array}$ $\begin{array}{lllllllllll}26 & \mathrm{~S} & 10 & \mathrm{MR} & \mathrm{S} & 4 & \mathrm{R} & 0 & \mathrm{R} & \mathrm{R}\end{array}$ $\begin{array}{llllllllll}85 & \mathrm{~S} & 20 & \mathrm{~S} & \mathrm{~S} & 14 & \mathrm{MS} & 0 & \mathrm{R} & \mathrm{MR}\end{array}$ $\begin{array}{llllllllllllllllll}4 & \mathrm{R} & 4 & \mathrm{R} & \mathrm{R} & 0 & \mathrm{R} & 0 & \mathrm{R} & \mathrm{R}\end{array}$ $\begin{array}{lllllllllll}22 & \mathrm{~S} & 6 & \mathrm{MR} & \mathrm{MS} & 4 & \mathrm{R} & 0 & \mathrm{R} & \mathrm{R}\end{array}$ $\begin{array}{lllllllllll}90 & \mathrm{~S} & 70 & \mathrm{~S} & \mathrm{~S} & 25 & \mathrm{~S} & 0 & \mathrm{R} & \mathrm{MS}\end{array}$ $\begin{array}{lllllllllll}81 & \mathrm{~S} & 15 & \mathrm{MS} & \mathrm{S} & 8 & \mathrm{MR} & 0 & \mathrm{R} & \mathrm{R}\end{array}$ $\begin{array}{lllllllllll}32 & \mathrm{~S} & 11 & \mathrm{MS} & \mathrm{S} & 0 & \mathrm{R} & 0 & \mathrm{R} & \mathrm{R}\end{array}$ 
ATTILA/BABAX//PASTOR

$\begin{array}{lllllllllllll}\text { CMSS98Y03454T-040M-020Y-030M-0 } & 5 & \mathrm{R} & 2 & \mathrm{R} & \mathrm{R} & 0 & \mathrm{R} & 0 & \mathrm{R} & \mathrm{R}\end{array}$ 40SY-020SY- B

HAMAM-4

$\begin{array}{lllllllllllll}\text { ICW92-0477-1AP-1AP-4AP-1AP-0AP } & 3 & \mathrm{R} & 2 & \mathrm{R} & \mathrm{R} & 1 & \mathrm{R} & 0 & \mathrm{R} & \mathrm{R}\end{array}$ $-\mathbf{B}$

SEIF-7

$\begin{array}{lllllllllllll}\text { ICW94-0121-0L-1AP-2AP-3AP-030AP- } & 8 & \text { MR } & 35 & \text { S } & \text { S } & 4 & \text { R } & 0 & \text { R } & \text { R }\end{array}$ 0APS-050AP-0AP- B

FOW-2/DOVIN-2

$\begin{array}{llllllllllll}\text { ICW94-0305-3AP-3AP-030AP-0APS-3 } & 4 & \mathrm{R} & 5 & \mathrm{R} & \mathrm{R} & 0 & \mathrm{R} & 0 & \mathrm{R} & \mathrm{R}\end{array}$ AP-0APS- B

ATTILA-4

$\begin{array}{lllllllllll}\text { CM85836-4Y-0M-0Y-6M-0Y-4PZ-0Y-2 } & 3 & \mathrm{R} & 4 & \mathrm{R} & \mathrm{R} & 0 & \mathrm{R} & 0 & \mathrm{R} & \mathrm{R}\end{array}$ SJ-0Y-0AP- B

HUBARA-16

$\begin{array}{llllllllllll}\text { ICW94-0392-0L-3AP-3AP-2AP-0APS-0 } & 6 & \text { MR } & 7 & \text { MR } & \text { MR } & 0 & \text { R } & 0 & \text { R } & \text { R }\end{array}$ AP- B

NANJING

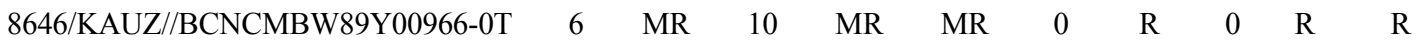
OPM-19Y-010M- B

OTUS

$\begin{array}{lllllllllll}\text { CMBW90Y3180-0TOPM-3Y-010M-01 } & 3 & \mathrm{R} & 5 & \mathrm{R} & \mathrm{R} & 0 & \mathrm{R} & 0 & \mathrm{R} & \mathrm{R}\end{array}$ $0 \mathrm{Y}-10 \mathrm{M}-015 \mathrm{Y}-0 \mathrm{Y}-1 \mathrm{KBY}-0 \mathrm{KBY}-\mathbf{B}$

SKAUZ*2/FCT

$\begin{array}{llllllllllll}\text { CMBW91M02703F-0TOPY-24M-010Y } & 48 & \mathrm{~S} & 40 & \mathrm{~S} & \mathrm{~S} & 0 & \mathrm{R} & 0 & \mathrm{R} & \mathrm{R}\end{array}$

$-010 \mathrm{M}-010 \mathrm{Y}-1 \mathrm{Y}-\mathbf{B}$

CBRD/KAUZ

CMSS94B00008S-0300M-0100Y-

0100M-18Y- 7M-0Y- 0HTY- B

SEKSAKA-11 CW94... B

ANGRA/2*CAZOCMBW90Y3215-0T

OPM-20Y

NESSER/SERI

CMSS93Y02623S-98Y-010Y-010Y-015

Y-4Y-05B-0Y-0SY-0AP- B

TJN/MV22- B

HD2329/SABUF

FPSS95B00253S-040Y-020M-040Y-020

Y-4M-0Y-0HTY- B

TJN/MV22- B

NEMURA/CETTIA

CMSW93Y00399S-1AP-2AP-1AP-0AP

S-0AP- B

CHAM-4- B

YUMAI 13/2*KAUZ- B

OPATA/RAYON//KAUZ

CMBW90Y2180-OTOPM-3Y-10M- B

$\begin{array}{lllllllllll}9 & \mathrm{MR} & 12 & \mathrm{MS} & \mathrm{MS} & 2 & \mathrm{R} & 0 & \mathrm{R} & \mathrm{R}\end{array}$

$\begin{array}{llllllllllll}6 & \mathrm{MR} & 13 & \mathrm{MS} & \mathrm{MR} & 1 & \mathrm{R} & 0 & \mathrm{R} & \mathrm{R}\end{array}$

$\begin{array}{lllllllllll}6 & \mathrm{MR} & 20 & \mathrm{~S} & \mathrm{MR} & 4 & \mathrm{R} & 0 & \mathrm{R} & \mathrm{R}\end{array}$

SITTA/CHIL//IRENA

$\begin{array}{llllllllllll}\text { CMBW91M03952T-0TOPY-6M-010SY } & 2 & \mathrm{R} & 4 & \mathrm{R} & \mathrm{R} & 0 & \mathrm{R} & 0 & \mathrm{R} & \mathrm{R}\end{array}$

$-015 \mathrm{M}-010 \mathrm{SY}-\mathbf{B}$

KOUHDASHT- B

LINE B- B

GAHAR- B

HAMAM-4

ICW92-0477-1AP-1AP-4AP-1AP-0AP-

$\begin{array}{cccccccccc}6 & \mathrm{MR} & 5 & \mathrm{R} & \mathrm{MR} & 0 & \mathrm{R} & 0 & \mathrm{R} & \mathrm{R} \\ 6 & \mathrm{MR} & 30 & \mathrm{~S} & \mathrm{~S} & 0 & \mathrm{R} & 0 & \mathrm{R} & \mathrm{R} \\ 1 & \mathrm{R} & 3 & \mathrm{R} & \mathrm{R} & 0 & \mathrm{R} & 0 & \mathrm{R} & \mathrm{R} \\ 3 & \mathrm{R} & 2 & \mathrm{R} & \mathrm{R} & 0 & \mathrm{R} & 0 & \mathrm{R} & \mathrm{R}\end{array}$


B

ZEMAMRA-8

$\begin{array}{lllllllllllll}\text { ICW91-0157-3AP-0TS-4AP-0TS-3AP-0 } & 12 & \text { MS } & 10 & \text { MR } & \text { MS } & 0 & \text { R } & 0 & \text { R } & \text { R }\end{array}$

L-0AP- B

CHEN/AEGILOPSSQUARROSA(TAU

S)//BCN/3/VEE\#7/BOW/4/PASTOR

CMSS93B01854T-040Y- B

CHEN/AEGILOPSSQUARROSA(TAU

S)//BCN/3/VEE\#7/BOW/4/PASTOR- B

SERI/RAYON

CRG2753.1-0B-099Y-099M-28Y-0B- B

TJN//GHK"S"/BOW"S"/3/SHIR- B

SITTA/CHIL/IRENA

CMBW91MO3952T-0TOPY-6M-010S

Y-015M- B

PIGO/PASTOR

$\begin{array}{llllllllllllll}\text { CMSS95M01046S-0100M-050SY-050 } & 25 & \mathrm{~S} & 12 & \mathrm{MS} & \mathrm{S} & 2 & \mathrm{R} & 0 & \mathrm{R} & \mathrm{R}\end{array}$

M-050SY-030M- B

BERKUT

$\begin{array}{llllllllllll}\text { CMSS96M05638T-040Y-26M-010SY-0 } & 19 & \mathrm{~S} & 10 & \mathrm{MR} & \mathrm{MS} & 4 & \mathrm{R} & 0 & \mathrm{R} & \mathrm{R}\end{array}$ 10M-010SY- B

SERI*3//RL6010/4*YR/3/PASTOR/4/B

AV92

CMSS96M05696T-040Y-14M-010SY-0

$\begin{array}{lllllllllll}6 & \text { MR } & 20 & \mathrm{~S} & \mathrm{MS} & 0 & \mathrm{R} & 0 & \mathrm{R} & \mathrm{R}\end{array}$

$\begin{array}{lllllllllll}26 & \mathrm{~S} & 35 & \mathrm{~S} & \mathrm{~S} & 4 & \mathrm{R} & 0 & \mathrm{R} & \mathrm{R}\end{array}$

$\begin{array}{llllllllllll}30 & \mathrm{~S} & 25 & \mathrm{~S} & \mathrm{~S} & 3 & \mathrm{R} & 0 & \mathrm{R} & \mathrm{R}\end{array}$

$\begin{array}{lllllllllll}9 & \mathrm{MR} & 6 & \mathrm{MR} & \mathrm{MR} & 0 & \mathrm{R} & 0 & \mathrm{R} & \mathrm{R}\end{array}$

$\begin{array}{lllllllllll}6 & \mathrm{MR} & 5 & \mathrm{R} & \mathrm{MR} & 1 & \mathrm{R} & 0 & \mathrm{R} & \mathrm{R}\end{array}$

10M- B

PASTOR/ /HXL 7573/2*BAU

$\begin{array}{llllllllllllllll}\text { CMSS97M00406S-0P20Y-97M-010Y- } & 25 & \text { S } & 30 & \text { S } & \text { S } & 0 & \text { R } & 0 & \text { R } & \text { R }\end{array}$

B

CROC_1/AE.SQUARROSA(213)//PGO

/3/BABAX CMSS97M00814S-030M- B

BAVIACORA M 92

$\begin{array}{llllllllllllll}\text { CM92066-J-OY-OM-OY-4M-OY-OME } & 11 & \text { MS } & 5 & \text { R } & \text { MR } & 1 & \text { R } & 0 & \text { R } & \text { R }\end{array}$

$\mathrm{X}-\mathbf{B}$

GHK"S"BOW"S"//90 -ZHONG87- B

KATILA-11- B

NESTOR/3/HE1/3*CNO79//2*SERI

$\begin{array}{llllllllllllllll}\text { CMSS92M00092S-015M-0Y-0Y-050M- } & 20 & \mathrm{~S} & 20 & \mathrm{~S} & \mathrm{~S} & 1 & \mathrm{R} & 0 & \mathrm{R} & \mathrm{R}\end{array}$

25Y-2M-0Y- B

SERI82/SHUHA "S"

$\begin{array}{lllllllllllll}\text { ICW89-0018-7AP-0AP-1AP-0TS-0AP- } & 12 & \text { MS } & 13 & \text { MS } & \text { MS } & 4 & \text { R } & 0 & \text { R } & \text { R }\end{array}$

B

FLORKWA-2/KAUZ

ICW94-0392-0L-392-0L- B

FLORKWA-2/KAUZ

ICW94-0392-0L-3AP-2AP- B

FLORKWA-2/KAUZ

ICW94-0392-0L-3AP-3AP- B

SAMAR-2

$\begin{array}{lllllllllllll}\text { CM112793-OTOPY-22M-020Y-0LO0M } & 13 & \text { MS } & 6 & \text { MR } & \text { MR } & 1 & \text { R } & 0 & \text { R } & \text { R }\end{array}$

-1Y-010M-10Y-0M-0AP- B

$\begin{array}{llllllllllll}\text { PREWCM59377-3AP-2AP-1AP-0AP-B } & 12 & \text { MS } & 2 & \text { R } & \text { MR } & 0 & \text { R } & 0 & \text { R } & \text { R }\end{array}$

BABAGA-1

ICW93-006S-8AP-0L-1AP-0L-0AP- B

NADIA- B

VEE\#8//JUP/BJY/3/T3.71/TRM/4/BCN/

5/ KAUZCMB W89Y01242-

$\begin{array}{llllllllllll}16 & \mathrm{~S} & 10 & \mathrm{MR} & \mathrm{MS} & 4 & \mathrm{R} & 0 & \mathrm{R} & \mathrm{R}\end{array}$

$\begin{array}{lllllllllll}12 & \mathrm{MS} & 2 & \mathrm{R} & \mathrm{MR} & 0 & \mathrm{R} & 0 & \mathrm{R} & \mathrm{R}\end{array}$

$\begin{array}{llllllllll}7 & \mathrm{MR} & 6 & \mathrm{MR} & \mathrm{MR} & 3 & \mathrm{R} & 0 & \mathrm{R} & \mathrm{R}\end{array}$

$\begin{array}{lllllllllllllllll}7 & \mathrm{MR} & 3 & \mathrm{R} & \mathrm{R} & 0 & \mathrm{R} & 0 & \mathrm{R} & \mathrm{R}\end{array}$

$\begin{array}{lllllllllll}7 & \mathrm{MR} & 0 & \mathrm{R} & \mathrm{R} & 3 & \mathrm{R} & 0 & \mathrm{R} & \mathrm{R}\end{array}$

$\begin{array}{lllllllllll}8 & \mathrm{MR} & 0 & \mathrm{R} & \mathrm{R} & 0 & \mathrm{R} & 0 & \mathrm{R} & \mathrm{R}\end{array}$ 
0T0PM-29Y- B

87 ZHONG 291- B

CHAM-H/KASGLE- B

SIHA/CHIL//IRENA- B

QAFZEH-33- B

ATRIS- B

IZAZ-21CW92-0671-4AP-0L-4AP-0L-1

AP-0AP- B

CHORIZO/BOCRO-4

ICW93-0566-3AP-0L-1AP- B

KASYON/GEUARO 81//TEVEE-1

$$
\text { ICW92- B }
$$

SHUHA-17/SHUHA-18- B

\section{CHAM-6- B}

PASTOR- B

PASTOR *'2/OPATA- B

BANA-4- B

ANGI-2- B

ZAGROS- B

E90040/MFOWL'13CDSS93Y35-1Y-1

Y-OB-OY-2B-OY- D

SRN 1/LARU/3/YAV

/FGO//ROH/4/LICAN- D

TANTLO//CREX/ALLA/3TANTLO

CD99528-E-2PAP- D

ZEGZAG/ALTAR 84//DIPPER 2

CDSS93Y1347-A-1Y-1Y-0B- D

31-19-2-2- D

18-18-1-4- D

43-25-2-4- D

Arislahn-4- D

Lgt3/4/Bcr/3/Chi//Gta/Stk- D

Bcr//Memo/goo- D

Bcr//Memo/goo/3/Stjy- D

D68-1-93A-1A//Ruff/Fg/3/Mtl-5/4/Lahn-

D

Terbo 167-3- D

Bcr//fg/snbipe/3/Gdovz

578/swan//Ddra2- D

Fadda-98

Villemur/3/Lahn//gs/stk/4/Dra2/Bcr- D

Terbo 197-4- D

Anonymous- D

Anonymous - D

Anonymous - D

Anonymous - D

Anonymous - D

Anonymous - D

Anonymous - D

Stj3//Bcr/LKS4- D

Saimarae- D

Bigost-1

ICD96-0887-C-2AP-0AP-5AP-0AP- D $\begin{array}{cccccccccc}5 & \mathrm{R} & 0 & \mathrm{R} & \mathrm{R} & 0 & \mathrm{R} & 0 & \mathrm{R} & \mathrm{R} \\ 3 & \mathrm{R} & 3 & \mathrm{R} & \mathrm{R} & 3 & \mathrm{R} & 0 & \mathrm{R} & \mathrm{R} \\ 8 & \mathrm{MR} & 4 & \mathrm{R} & \mathrm{MR} & 0 & \mathrm{R} & 0 & \mathrm{R} & \mathrm{R} \\ 90 & \mathrm{~S} & 40 & \mathrm{~S} & \mathrm{~S} & 5 & \mathrm{R} & 0 & \mathrm{R} & \mathrm{R} \\ 10 & \mathrm{MR} & 9 & \mathrm{MR} & \mathrm{MR} & 0 & \mathrm{R} & 0 & \mathrm{R} & \mathrm{R} \\ 15 & \mathrm{MS} & 16 & \mathrm{~S} & \mathrm{~S} & 0 & \mathrm{R} & 0 & \mathrm{R} & \mathrm{R}\end{array}$

$\begin{array}{lllllllllllllllllllll}4 & \mathrm{R} & 4 & \mathrm{R} & \mathrm{R} & 0 & \mathrm{R} & 0 & \mathrm{R} & \mathrm{R}\end{array}$

$\begin{array}{llllllllllllllllllll}30 & \mathrm{~S} & 20 & \mathrm{~S} & \mathrm{~S} & 0 & \mathrm{R} & 0 & \mathrm{R} & \mathrm{R}\end{array}$

$\begin{array}{llllllllll}10 & \mathrm{MR} & 10 & \mathrm{MR} & \mathrm{MR} & 4 & \mathrm{R} & 0 & \mathrm{R} & \mathrm{R}\end{array}$

$\begin{array}{llllllllll}21 & \mathrm{~S} & 11 & \mathrm{MS} & \mathrm{S} & 1 & \mathrm{R} & 0 & \mathrm{R} & \mathrm{R}\end{array}$

$\begin{array}{llllllllll}16 & \mathrm{~S} & 15 & \mathrm{MS} & \mathrm{S} & 1 & \mathrm{R} & 0 & \mathrm{R} & \mathrm{R}\end{array}$

$\begin{array}{llllllllll}24 & \mathrm{~S} & 30 & \mathrm{~S} & \mathrm{~S} & 4 & \mathrm{R} & 0 & \mathrm{R} & \mathrm{R}\end{array}$

$\begin{array}{llllllllll}28 & \mathrm{~S} & 25 & \mathrm{~S} & \mathrm{~S} & 2 & \mathrm{R} & 0 & \mathrm{R} & \mathrm{R}\end{array}$

$\begin{array}{llllllllll}19 & \mathrm{~S} & 20 & \mathrm{~S} & \mathrm{~S} & 2 & \mathrm{R} & 0 & \mathrm{R} & \mathrm{R}\end{array}$

$\begin{array}{llllllllll}41 & \mathrm{~S} & 30 & \mathrm{~S} & \mathrm{~S} & 0 & \mathrm{R} & 0 & \mathrm{R} & \mathrm{R}\end{array}$

$\begin{array}{llllllllll}41 & \mathrm{~S} & 30 & \mathrm{~S} & \mathrm{~S} & 0 & \mathrm{R} & 0 & \mathrm{R} & \mathrm{R} \\ 1 & \mathrm{R} & 0 & \mathrm{R} & \mathrm{R} & 0 & \mathrm{R} & 0 & \mathrm{R} & \mathrm{R}\end{array}$

ICAMOR-TA04-63 F4

13/3/Arthur71/Lahn//Blk2/Lahn/4/Quar mal ICD96- D 
ICAMOR-TA04-71 F4

13/3/Arthur71/Lahn//Blk2/Lahn/4/Quar mal ICD96- D

Tunsyr-1- D

ALTAR84/BISU-1//BUSCA-3- D

Ammar-10

$\begin{array}{llllllllllll}\text { ICD94-0918-C-12AP-0AP-6AP-0AP-4A } & 12 & \text { MS } & 0 & \text { R } & \text { MR } & 0 & \text { R } & 0 & \text { R } & \text { R }\end{array}$ P-0AP- D

Geruftel-1

$\begin{array}{lllllllllllll}\text { ICD95-1302-C-3AP-0AP-1AP-0AP-5AP } & 0 & \mathrm{R} & 0 & \mathrm{R} & \mathrm{R} & 0 & \mathrm{R} & 0 & \mathrm{R} & \mathrm{R}\end{array}$

- D

MEXI75//YAV-10/AUK- D

ARMENT//SRN-3/NIGRIS-4/3/CANEL

O-9.1- D

SOMAT-4/INTER-8- D

PLATA-1/SNM//PLATA-9/3/TARRO-3

- D

SRN-1/KILL//2*FOLTA-1- D

GREEN-14//YAV-10/AUK- D

GA//2*CHEN/ALTAR84- D

$\mathrm{BCR} / \mathrm{MEMO} / \mathrm{GOO} / 3 / \mathrm{STJ} 7-\quad$ D

SERRATOR-1//SRN-3/AJAIA-15- D

D68-1-93A-1A//Ruff/Fg/3Mtl-5/4/Lahn-

D

D68-1-93A-1A//Ruff/Fg/3Mtl-5/4/Lahn-

D

GREEN-14//YAV-10/AUK- D

Bisu-1//CHEN-1/TEZ/3/HUI//CIT71/Cll

- D

$\mathrm{Bcr} / 3 / \mathrm{Ch} 1 / / \mathrm{Gta} / \mathrm{Stk} / 4 / \mathrm{Bcr} / \mathrm{Lks} 4 \mathrm{ICD} 92-01$

50-Cabl -11AP-0AP- D

Gsb1-1/4/D68/1/93A-1A//Ruff/Fg/3/Mtl-

5I CD95- D

Altar84/Stn/Wdz-2ICD92-MABL-0238-

4AP-0AP-5AP- D

DON-Md 81-36- D

Stj3//Bcr/Lks4- D- D

Stj//Bcr/LKS41CD94- D

OUASERL-1

ICD96-0758-C-2AP-0AP-5AP-0AP- D
TRE97/4/GdoVz512/CIT/RUFF/FG/3/E

NTE/MARIO//C- D

$\begin{array}{llllllllll}0 & \mathrm{R} & 0 & \mathrm{R} & \mathrm{R} & 0 & \mathrm{R} & 0 & \mathrm{R} & \mathrm{R} \\ 4 & \mathrm{R} & 1 & \mathrm{R} & \mathrm{R} & 0 & \mathrm{R} & 0 & \mathrm{R} & \mathrm{R} \\ 3 & \mathrm{R} & 1 & \mathrm{R} & \mathrm{R} & 0 & \mathrm{R} & 0 & \mathrm{R} & \mathrm{R}\end{array}$

MARSYR-6

ICD95-1127-T-0AP-9AP-0AP-7AP-0AP

- D

ETH-LRBRA1-133/3*ALTER84

CDSP91 B931-A-1H-030Y-030M-3Y- D

21563/AA//Fg/3/D68-10-2A- D

CARGO-8//SORA/PLATA-12- D

KIRKI-1/HIMAN-9- D

Massara-1/4/Aus1/3/Scar/Gdovz579//Bit

Agar1/5/shea/stk//bit/3/kyp/4/chah88- D

Gdrl/BEZAIZ-SHF//Aznl- D

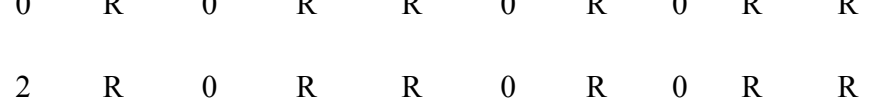

$\begin{array}{llllllllllllllll}0 & \mathrm{R} & 0 & \mathrm{R} & \mathrm{R} & 1 & \mathrm{R} & 0 & \mathrm{R} & \mathrm{R}\end{array}$

$\begin{array}{lllllllllll}6 & \mathrm{MR} & 0 & \mathrm{R} & \mathrm{R} & 0 & \mathrm{R} & 0 & \mathrm{R} & \mathrm{R}\end{array}$

$\begin{array}{llllllllllll}0 & \mathrm{R} & 0 & \mathrm{R} & \mathrm{R} & 0 & \mathrm{R} & 0 & \mathrm{R} & \mathrm{R}\end{array}$

$\begin{array}{llllllllll}2 & \mathrm{R} & 0 & \mathrm{R} & \mathrm{R} & 0 & \mathrm{R} & 0 & \mathrm{R} & \mathrm{R}\end{array}$

$\begin{array}{llllllllll}1 & \mathrm{R} & 0 & \mathrm{R} & \mathrm{R} & 0 & \mathrm{R} & 0 & \mathrm{R} & \mathrm{R}\end{array}$

$\begin{array}{llllllllll}5 & \mathrm{R} & 0 & \mathrm{R} & \mathrm{R} & 0 & \mathrm{R} & 0 & \mathrm{R} & \mathrm{R}\end{array}$

$\begin{array}{llllllllll}6 & \mathrm{MR} & 2 & \mathrm{R} & \mathrm{R} & 0 & \mathrm{R} & 0 & \mathrm{R} & \mathrm{R}\end{array}$

$\begin{array}{llllllllll}2 & \mathrm{R} & 0 & \mathrm{R} & \mathrm{R} & 0 & \mathrm{R} & 0 & \mathrm{R} & \mathrm{R}\end{array}$

$\begin{array}{lllllllllll}4 & \mathrm{R} & 0 & \mathrm{R} & \mathrm{R} & 0 & \mathrm{R} & 0 & \mathrm{R} & \mathrm{R}\end{array}$

$\begin{array}{llllllllllll}5 & \mathrm{R} & 3 & \mathrm{R} & \mathrm{R} & 0 & \mathrm{R} & 0 & \mathrm{R} & \mathrm{R}\end{array}$

$\begin{array}{lllllllllllll}0 & \mathrm{R} & 0 & \mathrm{R} & \mathrm{R} & 0 & \mathrm{R} & 0 & \mathrm{R} & \mathrm{R}\end{array}$

$\begin{array}{lllllllllll}7 & \mathrm{MR} & 0 & \mathrm{R} & \mathrm{R} & 0 & \mathrm{R} & 0 & \mathrm{R} & \mathrm{R}\end{array}$

$\begin{array}{llllllllllll}4 & \mathrm{R} & 5 & \mathrm{R} & \mathrm{R} & 1 & \mathrm{R} & 0 & \mathrm{R} & \mathrm{R}\end{array}$

$\begin{array}{llllllllllll}0 & \mathrm{R} & 0 & \mathrm{R} & \mathrm{R} & 1 & \mathrm{R} & 0 & \mathrm{R} & \mathrm{R}\end{array}$

Miki-2

ICD94-0994-CABL-10AP-0AP-1AP-0A P- D 


\begin{tabular}{|c|c|c|c|c|c|c|c|c|c|c|}
\hline $\begin{array}{l}\text { Ossl-1/4/MrbSH/3/Rabi//Gs/Cr/5/Hna } \\
\text { ICD96-0744-C- D }\end{array}$ & 0 & $\mathrm{R}$ & 0 & $\mathrm{R}$ & $\mathrm{R}$ & 4 & $\mathrm{R}$ & 0 & $\mathrm{R}$ & \\
\hline $\begin{array}{l}\text { PLATA_1/SNM//PLATA_9 } \\
\text { CD97899-H-2Y-040M- D }\end{array}$ & 1 & $\mathrm{R}$ & 0 & $\mathrm{R}$ & $\mathrm{R}$ & 0 & $\mathrm{R}$ & 0 & $\mathrm{R}$ & $\mathrm{R}$ \\
\hline $\begin{array}{l}\text { RASCON_39/TILO_1 } \\
\text { SDSS92B611-2M-0Y-0M-0Y-1B-0Y- D } \\
\text { HUI/YAV79//DON87 }\end{array}$ & 2 & $\mathrm{R}$ & 0 & $\mathrm{R}$ & $\mathrm{R}$ & 1 & $\mathrm{R}$ & $c$ & $\mathrm{R}$ & $\mathrm{R}$ \\
\hline $\begin{array}{l}\text { CDSS93Y152-15Y-0M-0Y-0B-3Y-0B- } \\
\text { D }\end{array}$ & 0 & $\mathrm{R}$ & 2 & $\mathrm{R}$ & $\mathrm{R}$ & 0 & $\mathrm{R}$ & c & $\mathrm{R}$ & $\mathrm{R}$ \\
\hline $\begin{array}{l}\text { Saadi 1989/Chacan } \\
\text { ICD96-0163-T-0AP-1AP-0AP-4AP- D }\end{array}$ & 3 & $\mathrm{R}$ & 0 & $\mathrm{R}$ & $\mathrm{R}$ & 0 & $\mathrm{R}$ & c & $\mathrm{R}$ & $\mathrm{R}$ \\
\hline $\begin{array}{l}\text { DA-6 Black awns/3/Bcr//Memo/Goo } \\
\text { ICD96-0058-C-0AP-2AP- D }\end{array}$ & 2 & $\mathrm{R}$ & 0 & $\mathrm{R}$ & $\mathrm{R}$ & 0 & $\mathrm{R}$ & 0 & $\mathrm{R}$ & $\mathrm{R}$ \\
\hline $\begin{array}{l}\text { DA-6 Black awns/3/Bcr//Memo/Goo } \\
\text { ICD96-0058-C-0AP-2AP- D }\end{array}$ & 4 & $\mathrm{R}$ & 0 & $\mathrm{R}$ & $\mathrm{R}$ & 1 & $\mathrm{R}$ & 0 & $\mathrm{R}$ & $\mathrm{R}$ \\
\hline $\begin{array}{l}\text { Maamouri-2 } \\
\text { ICD94-0404-T-7AP-0AP-1AP-0AP- D } \\
\text { Arislahn-10 }\end{array}$ & 2 & $\mathrm{R}$ & 0 & $\mathrm{R}$ & $\mathrm{R}$ & 0 & $\mathrm{R}$ & c & $\mathrm{R}$ & $\mathrm{R}$ \\
\hline $\begin{array}{l}\text { ICD94-0422-T-3AP-0AP-2AP-0AP-4AP } \\
-0 \text { AP- D }\end{array}$ & 3 & $\mathrm{R}$ & 0 & $\mathrm{R}$ & $\mathrm{R}$ & 0 & $\mathrm{R}$ & 0 & $\mathrm{R}$ & $\mathrm{R}$ \\
\hline $\begin{array}{l}\text { Ter-1/3/Stj3//Bcr/Lks4ICD99-1036-T-O } \\
\text { AP-9AP-AP-1AP- D } \\
\text { Icasyr-2 }\end{array}$ & 0 & $\mathrm{R}$ & 0 & $\mathrm{R}$ & $\mathrm{R}$ & 0 & $\mathrm{R}$ & $c$ & $\mathrm{R}$ & $\mathrm{R}$ \\
\hline $\begin{array}{l}\text { ICD95-0169-C-OAP-2AP-0AP-4AP-OA } \\
\text { P- D }\end{array}$ & 1 & $\mathrm{R}$ & 0 & $\mathrm{R}$ & $\mathrm{R}$ & 0 & $\mathrm{R}$ & 0 & $\mathrm{R}$ & $\mathrm{R}$ \\
\hline Ammar-1 & & & & & & & & & & \\
\hline ICD94-0918-C-12AP-0AP-4AP-OAP-4 & 6 & MR & 16 & $\mathrm{~S}$ & MS & 0 & $\mathrm{R}$ & 0 & $\mathrm{R}$ & $\mathrm{R}$ \\
\hline
\end{tabular}

R: resistant, MR: moderately resistant, MS: moderately susceptible, $\mathbf{S}$ : susceptible, DI: disease index, PI: percentage of infection, MDI: mean of disease index, D: durum wheat, B: bread wheat.

Results of in 2008 showed that, among the 47 entries adapted to the cold regions, 23 showed resistant reaction. From lines of ARWYT, three were resistant and eight were susceptible and from 18 lines of URDYT, 15 were resistant. Three commercial cultivars, Zardak, Sardari and Aazr-2 were resistant, partially resistant and susceptible respectively. Assessment for 40 genotypes related to the preliminary yield trials revealed that 20 were resistant. Among the URBYT and URDYT entries, 29 lines were resistant; the also 37 lines without infection observed in lines of ERWYT 37 and in set of 19 lines of ARDYT, 18 lines were resistant. For all warm commercial cultivars, Saimarae, Gahar, Kouhdasht and Cham were resistant, Pastor was mediate-susceptible and Zagros was susceptible (Table 2).

\subsection{Reactions to T. controversa during 2007 and 2008}

Reaction to common bunt differed among genotypes in 2007. Among the 47 experimental lines adapted to cold regions, 18 showed resistance reaction. From 14 lines belong to ARWYT, 10 were susceptible and the rest were partially resistant. Among the 18 lines of URDYT, 17 were resistant and one was partially-resistance. For 40 test genotypes belong to preliminary yield trials 34 lines were resistant. For trials of URDYT and URBYT, only one line was susceptible and all lines of ARDYT and ERWYT were resistant. Cold commercial cultivars, Zardak, Sardari and Azar-2 did not show any infection. Of the six warm commercial cultivars, Gahar, Kouhdasht, saimarae, Cham-4, Pastor and Zagros all were resistant. In 2008, almost all 240 cultivars and lines had resistant reaction to $T$. controversa. As it will be explained further below, experimental condition was not favored disease in year of 2008 (Table 1).

\subsection{Comparison between years 2007 and 2008 for Genotypes Reaction to T. laevis}

Examined genotypes had different reactions against to T. laevis in 2007 and 2008 (Figure 1), nevertheless same lines were used. The greatest disease severity was observed in 2007 than 2008, as whole susceptible genotypes were about $26.66 \%$ and $19.16 \%$ in 2007 and 2008, respectively. Reaction of cold and warm commercial cultivars was almost similar in two successive years. In general, durum genotypes showed the most disease resistance than bread ones (Table 1 and Figures $3 \& 5$ ). 


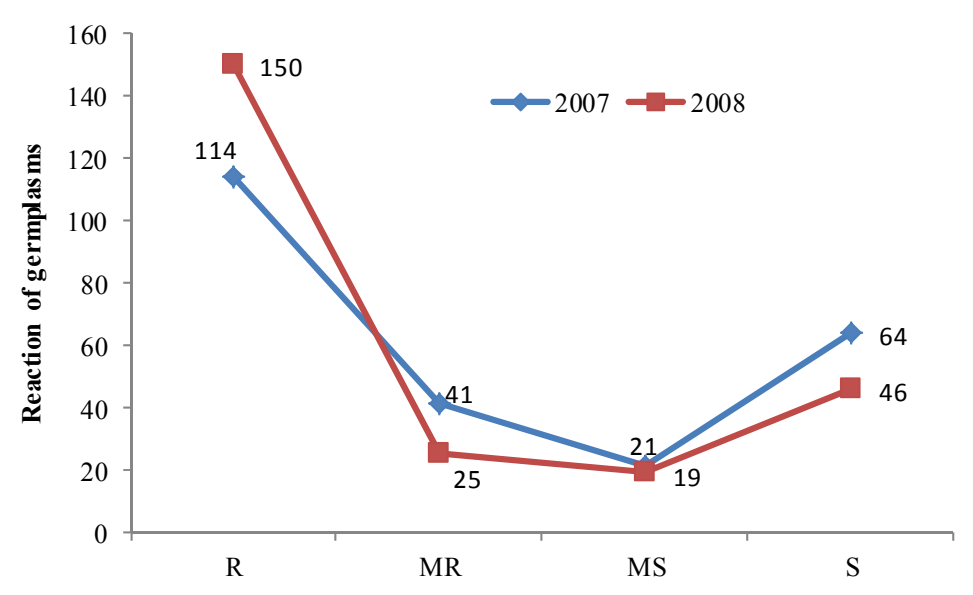

Figure 1. The comparison of the reaction of genotypes than common bunt in 2007 and 2008

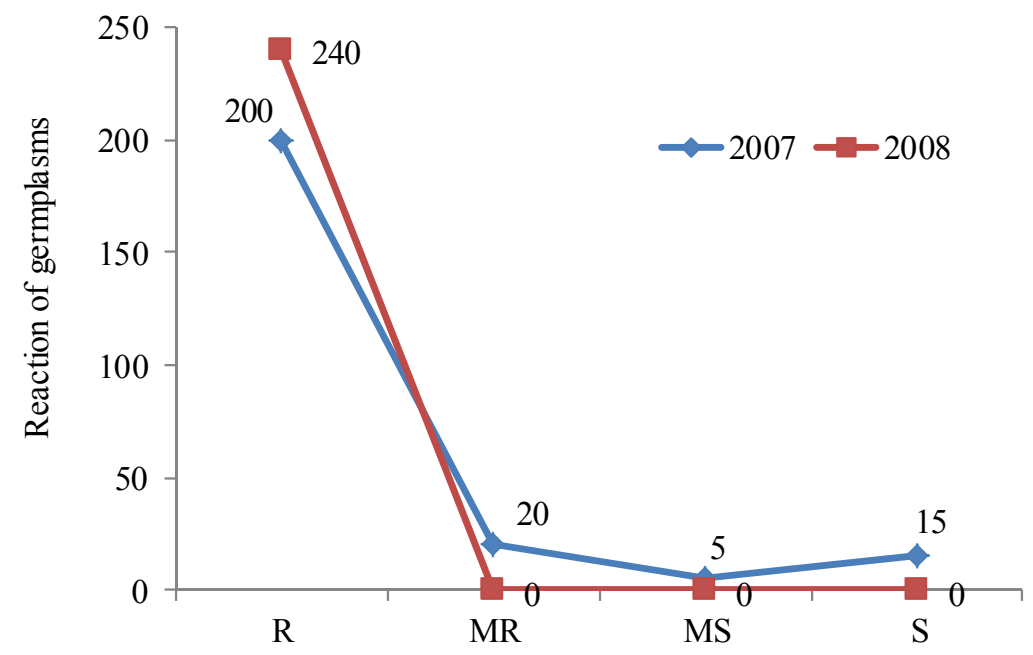

Figure 2. The comparison of the reaction of genotypes than dwarf bunt in 2007 and 2008

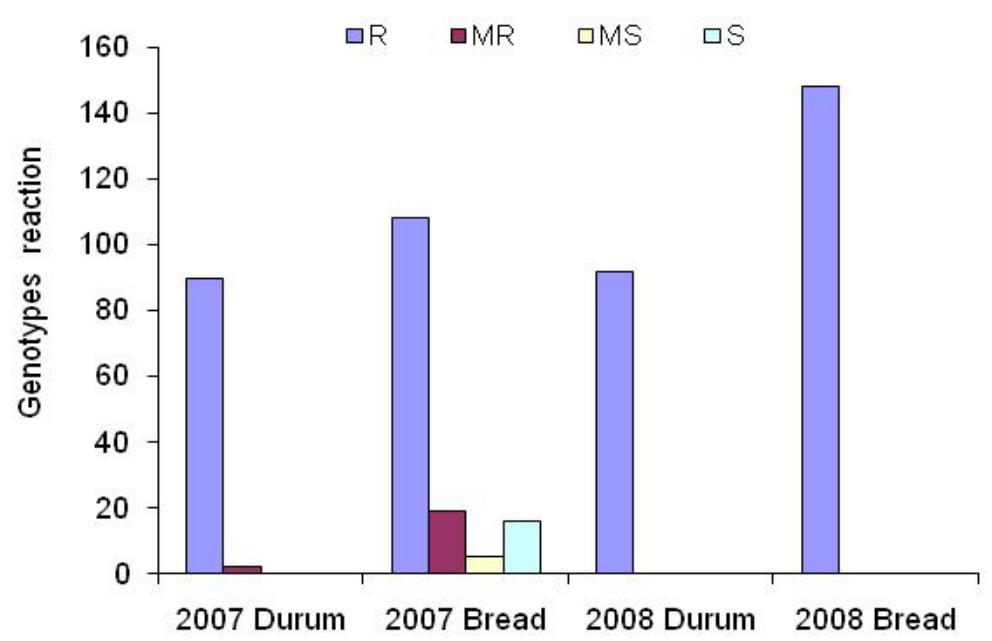

Figure 3. Reaction of Durum and Bread wheat genotypes to T. laevis during 2007 and 2008 


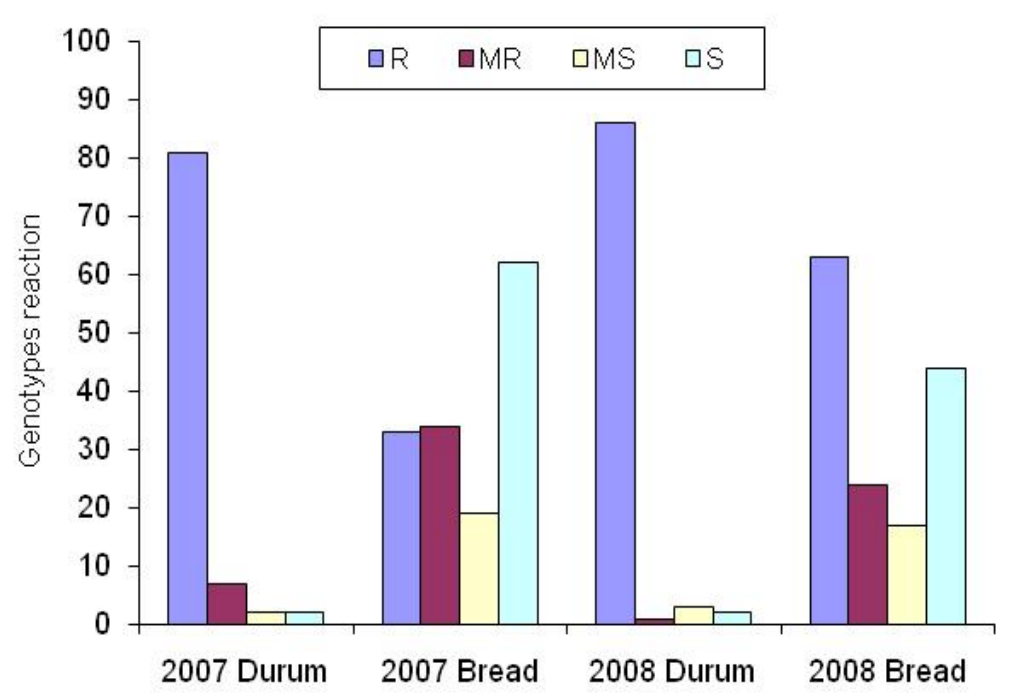

Figure 4. Reaction of Durum and Bread wheat genotypes to T. controversa during 2007 and 2008
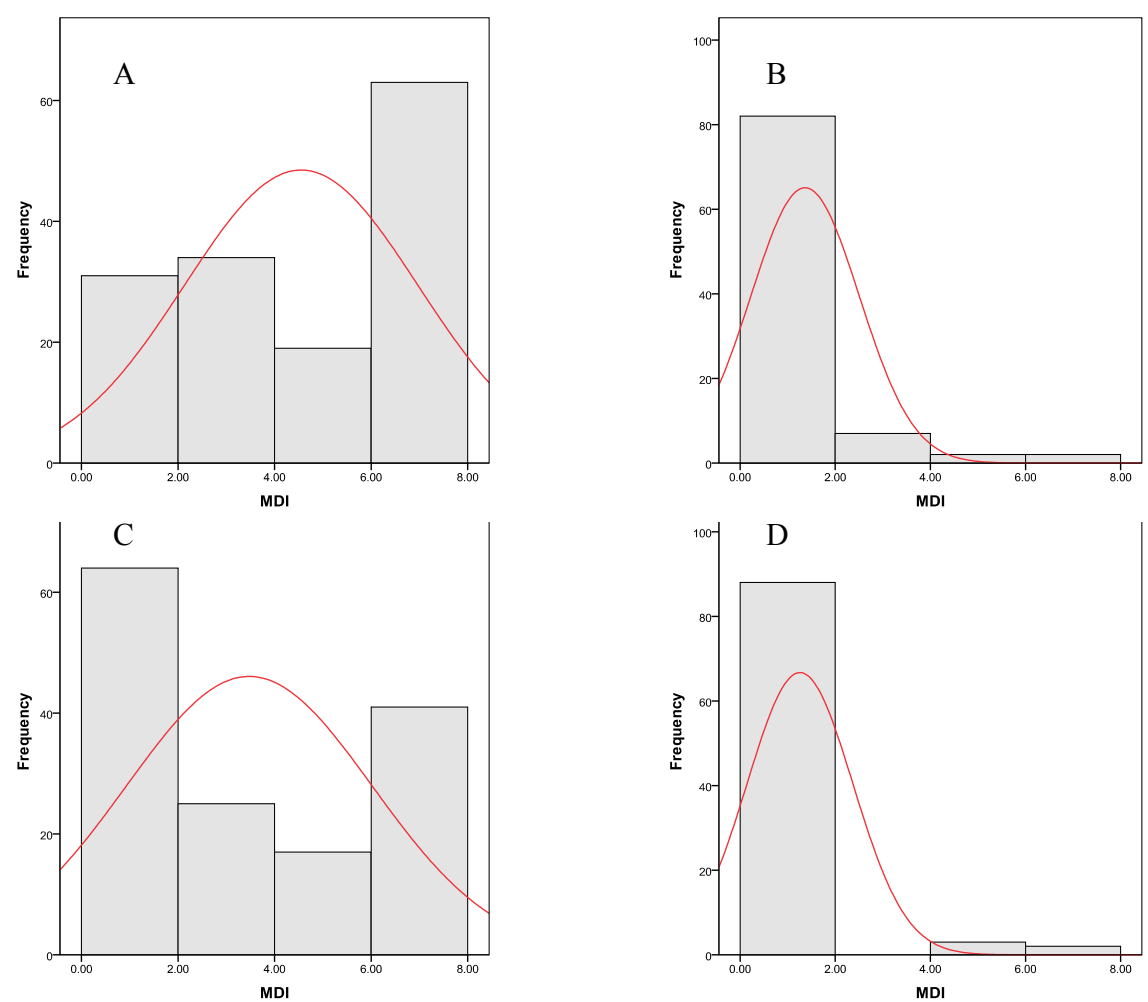

Figure 5. Distribution of 240 wheat genotypes displayed by their mean disease index (MDI) and frequencies at different situations, (A) Bread wheat in 2007; (B) Durum wheat in 2007; (C) Bread wheat in 2008 and (D) Durum wheat in 2008

\subsection{Comparison between Years 2007 and 2008 for Genotypes Reaction to T. controversa}

Responses to $T$. controversa were variable over two years. Eighty three percent of genotypes were resistant in 2007 but in 2008100 percent of them (Figure 2). Although reaction of cultivars and lines were greatly affected by climate of area in 2008, but recorded data of the first year, proved existing of high disease resistance among these genotypes. These results confirmed that resistance in durum genotypes is higher than bread wheat genotypes (Table 2 and Figures 4 \& 5). 
Paired $t$-test analysis showed significant differences between two experimental years for bread wheat genotypes exposed to $T$. laevis $(t=-5.53, \mathrm{P}<0.001)$ and also between two experimental years for bread wheat genotypes exposed to T. conroversa $(t=-6.39, \mathrm{P}<0.001)$. Paired $t$-test between two successive years of trial for durum wheat entries was not significant in relation to both pathogens. These results indicate that abiotic stresses are intensity and duration of stresses showing differences in cold tolerance between accessions (Table 3). With unequal variance assumptions, $t$-test analyses were accomplished between bread and durum wheat varieties, and there were significant differences between means for both $T$. laevis and $T$. controversa pathogens (Table 3 ).

Table 3. Comparison of two related fungi of smut diseases on two bread and durum wheat genotypes over two years 2007 and 2008 at Kurdistan, Iran

\begin{tabular}{lllllll}
\hline & & $\begin{array}{l}\text { Degree of } \\
\text { Freedom }\end{array}$ & $\begin{array}{l}\text { Standard } \\
\text { Deviation }\end{array}$ & $\begin{array}{l}\text { Standard } \\
\text { Error }\end{array}$ & Mean & \multirow{2}{*}{-value } \\
\hline T. laevis & Bread & 146 & 2.186 & 0.181 & 4.014 & $12.85^{* *}$ \\
& Durum & 92 & 1.042 & 0.108 & 1.312 & \\
& Paired B.W. over years & 146 & - & 0.194 & -1.075 & $-5.53^{* *}$ \\
& Paired D.W. over years & 92 & - & 0.088 & -0.108 & $-1.22^{\text {ns }}$ \\
& Bread & 146 & 1.002 & 0.083 & 1.531 & $5.79^{* *}$ \\
& Durum & 92 & 0.231 & 0.024 & 1.032 & \\
& Paired B.W. over years & 146 & - & 0.162 & -1.034 & $-6.39^{* *}$ \\
& Paired D.W. over years & 92 & - & 0.021 & -0.022 & $-0.99^{\text {ns }}$ \\
\hline
\end{tabular}

B.W. = Bread wheat; D. W. = Durum wheat;

Significant at $\mathrm{P}=0.01$ presented by $* *$, and $\mathrm{ns}=$ non-significant.

\section{Discussion}

In current study, we investigated reaction of bread and durum wheat germplasms to bunt incidence in the field experiments. These trials determined that durum germplasms had better reaction to common and dwarf bunts than germplasms of bread wheat. The rate of disease incidence in each two years of 2007 and 2008 for T. laevis was varied from 0 to $90 \%$ but the number of susceptible cultivars was higher in 2007. The same prediction for $T$. controversa showed a narrower variation from 0 to $65 \%$ in 2007, while any infection was observed in 2008 . Several investigators detailed similar results in their studies (Dumalasova \& Bartos; 2006 \& 2007a \& 2010). This variation may be due to a different pathological pattern in the inoculation used or environmental conditions (Dumalasova \& Bartos, 2010; Miczyński 1953).

Optimum infection of common bunt occurs when susceptible seeds grown in soil at $5^{\circ}$ to $10^{\circ} \mathrm{C}$ and only slight infection arise at $22^{\circ} \mathrm{C}$ (Purdy \& Kendrick, 1963). The distribution and incidence of dwarf bunt is highly correlated with snow conditions (Curtis et al., 2002). For instance, lack of enough rainfall in fall 2008 as well as predominate cold weather at planting time in the area of experiments, lack of suitable snow cover for long period in the region and especially failure to enough grow seeds in the fall and growth them in spring could be effective in reducing disease incidence (Table 1). According to the meteorological data presented in Table 1, it is possible that the lack of adequate rainfall during the fall 2008 be the most effective factor in decreasing incidence of dwarf bunt, because in this situation, growth of chickpea seedlings is very slow and tillering time which is the most sensitive stage to dwarf bunt is not coinciding with appropriate temperature $\left(5-8 \mathrm{C}^{\circ}\right)$ for teliospores budding (Hoffmann, 1982). In general, sowing time, resistance / susceptibility of cultivars, growing habit (spring or winter) and adequate snow cover are the most influential factors in developing of $T$. controversa (Mathre, 1996). This study demonstrated the impact of climatic conditions on disease occurrence of dwarf bunt, it seems that better understanding of biological aspects of dwarf bunt and using resistant genotypes could be beneficial in manage it efficiently, because unlike of common bunt, chemical control of dwarf bunt is not very consistent (Mathre, 1996).

In survey of these varieties against $T$. laevis observed that with being severe infection on susceptible cultivars and also differences were observed in infection rate of genotypes due to their reaction to the disease as a result of presence or absence of resistance gene or genes in investigated germplasms, it is possible to find these germplasms as good sources of resistance, especially when the lines reaction to cold climate of URDYT related to durum wheat lines (tetrahaploids) compared to ARWYT bread wheat lines, this qualification is in accordance with study of Dumalasova and Bartos (2010) (Figures 3 and 4). In our results susceptibility of genotypes to 
common bunt, particularly in bread wheat entries was higher than dwarf bunt. It is probably due to presence of specific genes in these genotypes that can presumably overcome virulence genes of common bunt. In some studies, a few commercial cultivars reported as susceptible and also some resistant varieties introduced such as Globus and Bill cultivars (Dumalasova \& Bartos, 2007b), it has also been observed in some genotypes in our study. The disease could be controlled if necessary, by replacing durum wheat instead of bread wheat in critical areas with high infection and also with regard to presence of resistant genes among these germplasms and due to their desirable agronomic traits can be use as a donor of resistance genes in plant improvement programs.

\section{Conclusion}

In our study has showed that reaction of registered cold and warm cultivars was about similar in each two consecutive years rather to each two pathogens, this process may be indicative of a real reaction cultivars and genotypes than these pathogens. Its appears that apply of resistant germplasms and seed treatment by suitable fungicide can eradicate the inoculums of these two smuts, provided that with correct management prevented from breaking resistance.

\section{Reference}

Akbari, A., \& Zolghadri, M. (1988). Current status of crop production research, infrastructure and constraint in Iran. In Srivastava, J.P., Saxena, M. C., Varma, S., \& Tahir, M. (Eds.), Winter cereals and food legumes in the mountainous area (pp. 181-189). Syria.

Anonymous. (2010). Statistical database. Available at http://www.fao.org

Curtis, B. C., Rajaram, S., \& Gómez Macpherson, H. (2002). Bread wheat, improvement and production. FAO Plant Production and Protection Series, No, 30. http://www.fao.org/docrep/006/y4011e/y4011e00.htm

Dumalasová, V., \& Bartoš, P. (2007a). Reaction of spring wheat cultivars to common bunt caused by Tilletia tritici (Bjerk.) Wint. and Tilletia laevis (Kühn). Czech. J. Genet. Plant. Breedi, 43(3), 82-86.

Dumalasová, V., \& Bartoš, P. (2010). Reaction of wheat, alternative wheat and triticale cultivars to common bunt. Czech. J. Genet. Plant. Breed, 46(1), 14-20.

Dumalasová, V., \& Bartoš, P. (2006). Resistance of winter wheat cultivars to common bunt, Tilletia tritici (Bjerk.) Wint. and Tilletia laevis Kühn. J. Plant. Dis. Protect, 113(4), 159-163.

Dumalasová, V., \& Bartoš, P. (2007b). Reaction of winter wheat cultivars to common bunt Tilletia tritici (Bjerk.) Wint. and T. laevis Kühn. Plant. Protect. Sci, 43(4), 138-141.

Fisher, G. W., \& Holton, C. S. (1957). Biology and control of the smut fungi. New York, Ronald Press Company. 662. http://dx.doi.org/10.2307/2483223

Hoffmann, J. A. (1982). Bunt of wheat. Plant Dis., 66, 979-987. http://dx.doi.org/10.1094/PD-66-979

Kendrick, E. L., Metzger, R. J., \& Rohde, C. R. (1957). A possible new source of high resistance to wheat smut. Phytopathology, 47, 19.

Knox, R. E., Thommas, J. B., Depaum, R. M., Demeke, T. T., Laroch, A., \& Gaudal. D. A. (1998). Registation of common bunt resistance wheat germplasm Lines P8913-V2A5 P8917B4D4 and P8921-Q4C5. Crop Sci, 38(2), 569-570. http://dx.doi.org/10.2135/cropsci1998.0011183X003800020096x

Mamluk, O. F., \& Van Slageren, M. W. (1993). Resistance to common bunt, yellow rust, Leaf rust Septoria tritici blotch in wild einkorn and wild emmer wheat. Phytopathol Mediterr, 32, 14-19.

Mamluk, O. F. (1998). Bunts and smuts of wheat in North Africa and the Near East. Euphytica, 100(1-3), 45-50. http://dx.doi.org/10.1023/A:1018343603827

Mardoukhi, V. (1989). Laboratory and farm method of distinguishing Tilletia controversa cause of dwarf bunt and its control. Abstract cereal research seminar, Seed and Plant Improvement Institue. pp. 64-67.

Mathre, D. E. (1996). Dwarf bunt: politics, identification, and biology. Ann. Rev. Phytopathol, 34, 67-85. http://dx.doi.org/10.1146/annurev.phyto.34.1.67

Mcintosh, R. A. (1998). Breeding wheat for resistance to biotic stresses. Euphytica, 100, 19-34. http://dx.doi.org/10.1023/A:1018387402918

Metzger, R. J., Rhode, C. R., \& Hoffmann, J. A. (1977). Inheritance of resistance to common bunt in Triticum aestivum PI 178383. Agronomy, 64. 
Miczyński, K. (1956). Wrażliwość odmian pszenicy jarej na śnieć cuchnącą (Tilletia careis Tul.) przy sztucznym zakażeniu. Acta Agrobot, 5, 139-145.

Purdy, L. H., \& Kendrick, E. L. (1963). Influence of environmental factors on the development of wheat bunt in the pacific North West. VI. Effect of soil temperature and moisture on infection by soil spores. Phytopathology, 53, 416-418.

Rajaram, S., \& Van Ginkel, M. (1996). A guide to the CIMMYT bread wheat section. In Wheat Special Report No. 5. Mexico, DF, CIMMYT.

Ruzgas, V., \& Liatukas, Z. (2009). Response of Lithuanian winter wheat advanced lines to common bunt (Tilletia tritici (bjerk.) wint). Proc. Latvian. Acad. Sci, 63, 51-56. http://dx.doi.org/10.2478/v10046-0090010-0

Sharifnabi, B., \& Hejadroude, G. H. A. (1992). Occurrence and geographic distribution of Tilletia species attacking winter wheat in west and northwest of Iran. Iran. J. Plant. Pathol, 28, 85-96 (in Farsi). http://www.agrisis.org 